This document is confidential and is proprietary to the American Chemical Society and its authors. Do not copy or disclose without written permission. If you have received this item in error, notify the sender and delete all copies.

\title{
Electrically Switchable and Permanently Stable Light Scattering Modes by Dynamic Fingerprint Chiral Textures
}

\begin{tabular}{|r|l|}
\hline Journal: & ACS Applied Materials \& Interfaces \\
\hline Manuscript ID & am-2015-12854u.R2 \\
\hline Manuscript Type: & Article \\
\hline Date Submitted by the Author: & $30-$ Mar-2016 \\
\hline Complete List of Authors: & $\begin{array}{l}\text { Cheng, Ko-Ting; National Central University, Department of Optics and } \\
\text { Photonics } \\
\text { Lee, Po-Yi; National Central University, Department of Optics and Photonics } \\
\text { Qasim, Malik M.; University of Cambridge } \\
\text { Liu, Cheng-Kai; National Central University, Department of Optics and } \\
\text { Photonics } \\
\text { Cheng, Wen-Fa; National Central University, Department of Optics and } \\
\text { Photonics } \\
\text { Wilkinson, Timothy; University of Cambridge }\end{array}$ \\
\hline
\end{tabular}

SCHOLARONE ${ }^{\text {Mx }}$

Manuscripts 


\section{Electrically Switchable and Permanently Stable Light Scattering} Modes by Dynamic Fingerprint Chiral Textures

Ko-Ting Cheng ${ }^{1 *}$, Po-Yi Lee ${ }^{1}$, Malik M. Qasim ${ }^{2}$, Cheng-Kai Liu ${ }^{1}$, Wen-Fa Cheng ${ }^{1}$, and Timothy D. Wilkinson ${ }^{2}$

${ }^{1}$ Department of Optics and Photonics, National Central University, Taoyuan City 320, Taiwan

${ }^{2}$ Centre of Molecular Materials for Photonics and Electronics, Department of Engineering, University of Cambridge, $9 \mathrm{JJ}$ Thomson Avenue, Cambridge, CB3 OFA, United Kingdom

*Corresponding authors: chengkt@dop.ncu.edu.tw

ABSTRACT: Negative dielectric nematic liquid crystals (LCS) doped with two azobenzene materials provide electrically switchable and permanently stable scattering mode light modulators based on dynamic fingerprint chiral textures (DFCT) with inhomogeneously helical axes. These light modulators can be switched between transparent (stable large domains of DFCT) states and scattering (stable small domains of DFCT) states by applying electric fields with different frequencies. The generation of DFCT results from the long flexible side chains of the doped chiral dopant. That is, if the DFCT can be obtained, then the large domains of DFCT reflect an intrinsically stable state. Moreover, the stabilization of the small domains of DFCT are caused by the terminal rigid restricted side chains of the other doped chiral dopant. Experimentally, the 
required amplitude to switch the light modulator from a scattering (transparent) state to a transparent (scattering) state decreases as the frequency of the applied electric field increases (decreases) within the set limits. This study is the first report on the advantages of the light scattering mode of DFCT, including low operating voltage, permanently stable transmission, wide viewing angle, high contrast, and polarization-independent scattering and transparency.

KEYWORDS: liquid crystal, bistability, dynamic scattering, cholesterics, fingerprint texture, light modulator 


\section{Introduction}

In 1962, Richard Williams, a researcher working for RCA Corporation, proposed an effect based on electro-hydrodynamic instability to generate Williams' domains inside liquid crystals (LCs). In general, the electro-hydrodynamic flow induced by ionic motion caused by conductivity anisotropy results in instability. ${ }^{1}$ In 1968, G. H. Heilmeier, the leader of David Sarnoff Research Center of RCA, was the first to report this dynamic scattering effect in LCs. The world's first electronically controllable LC display was demonstrated on the basis of dynamic scattering mode (DSM) by Heilmeier et al. ${ }^{2}$ LC cell preparations, such as LC materials with a negative dielectric anisotropy $(\Delta \varepsilon<0)$, homeotropic alignment layers, ionic dopant to increase the conductivity, and application of DC or low-frequency AC electric field, are necessary to achieve light scattering based on DSM. The driving mechanism of DSM suggests that the anchoring force parallel to the substrates caused by the applied electric field onto negative dielectric nematic LCs can be combined with the anchoring force perpendicular to the substrates provided by the coated homeotropic alignment layer, and the applied electric field-induced ion transport to initially generate Williams' domains under applied DC or low-frequency AC electric fields perpendicular to the substrates. Furthermore, LCs become turbulent domains presenting a white and strong scattering effect. The scattering performance of DSM can be enhanced and grayscale can be observed by increasing the applied voltages. The scattering state can be reverted to a transparent state if the applied electric voltage is removed. The operating DC electric field to obtain the saturated contrast ratio of 
approximately 20 ranged from $40 \mathrm{kV} / \mathrm{cm}$ to $50 \mathrm{kV} / \mathrm{cm}(4-5 \mathrm{~V} / \mu \mathrm{m}){ }^{2}{ }^{2}$ As a result, DSM-based transmissive and reflective LC displays become unsuitable for practical electronics, especially those with extremely high operating voltage, large power consumption, unstable transmission and scattering, and low contrast. Despite these disadvantages, LC light modulation based on DSM does not require any polarizer for these modulators so that DSM is definitely substantial and worth to be promoted.

To overcome the problem shown above, Heilmeier et al. reported an electric field-controlled reflective optical storage effect on mixed nematic-cholesteric LC systems; the effect presents a scattering state when a DC or low-frequency AC electric field is applied. However, the scattering state gradually fades with time when the applied DC field is removed; this phenomenon suggests that the scattering is a quasi-stable state, not a permanently stable state. ${ }^{3}$ Furthermore, the scattering state can be rapidly reverted to its initial transparent state by applying a high frequency AC electric field with a high amplitude. Although the performance has been slightly improved, these disadvantages of light modulators based on DSM are accounted for the lack of practical LC device applications; these disadvantages also impede further research and development of LC devices. Moreover, electrically induced scattering textures in smectic A (SmA) phase LCs with a positive dielectric anisotropy were reported in $1978 .{ }^{4}$ The mechanisms of electrical writing and erasing information involve a turbulent scattering state resembling DSM for nematics and a dielectric reorientation of the LC molecules, respectively. This LC device can store the written information without a sustaining 
voltage and can electrically erase the information. Coles et al. reported electrically switchable bistable/multistable scattering LC devices by using SmA LCs. ${ }^{5,6}$ These multistable SmA-based LC devices have been used to observe coherent light emission. ${ }^{7,8}$ An optically opaque state is generated by applying a low-frequency voltage; as a result, doped ionic materials can move. By contrast, a haze-free transparent state is obtained by applying a high-frequency voltage; thus, ionic conduction is restrained. Grayscales can also be formed through the application of fields with different frequencies and/or amplitudes. Guo et al. also demonstrated bistable SmA LC devices based on polymer wall and polymer-dispersed LC (PDLC). ${ }^{9-10}$ Therefore, a high operating voltage is a crucial disadvantage in the practical applications of scattering mode LC light shutters based on nematic, SmA, and mixed nematic-cholesteric LCs. In addition to the proposed LC materials used to design scattering mode LC light shutters, PDLC is a good candidate to develop light shutters. PDLC presents several advantages, including high scattering state, electrically switchable property (voltage-off: scattering; voltage-on: transparency), and simple fabrication processes; thus, PDLC can be used for practical electronic applications. However, the operating voltage of a traditional PDLC light shutter is very high because of the anchoring force from the polymer wall. As such, scientists are trying to reduce the voltage and achieve a bistable state. ${ }^{11-13}$ Another issue for consideration is the narrow viewing angle of PDLC in a transparent state; this condition is caused by the viewing-angle-dependent refractive index of LCs.

To conserve energy and reduce power consumption, researchers have relied 
on bistable liquid crystal (LC) devices/displays, which do not require real-time information update and do consume power when the displayed image content is changed. ${ }^{5,6,13-15}$ In this study, we demonstrate the electrical switching (low voltage at different frequencies) of a DSM light modulator between scattering and transparent states. Moreover, we present the permanent stabilization of these two states on the basis of negative dielectric nematic LCs doped with two new azobenzene materials. The transparency and scattering performances of the LC light modulator are dependent on the domain sizes of the generated fingerprint textures with inhomogeneously helical axes parallel to the substrates. These fingerprint textures are called dynamic fingerprint chiral textures (DFCT). In addition, grayscales can be obtained electrically on the basis of the area ratio of the scattering and transparent textures. Following the procedure described in Ref. 3, we synthesized in-house two chiral azobenzene dopants, namely, QM-02-75 and QM-02-77, whose chemical structures are shown in Figures 1a and 1b, respectively, on the basis of bis(azo) dopants to achieve stable dynamic scattering mode. Intrinsically, the synthesized bis(azo) materials, whose bases were first reported by Li et al., are axially chiral dopants with a high helical twisting power (HTP). ${ }^{16}$ This kind of chiral bis(azo) dopants is employed as a light-driven azobenzene material. ${ }^{17-23}$ Among the chemical structures of our synthesized materials, the binaphthyl core in the center provides high chirality to the RR or SS molecule. The $\mathrm{N}$-azo linkage is adopted to increase the solubility of binaphthyl chiral dopants into LCs that possess negative dielectric anisotropy. The properties of our synthesized bis(azo) compound, QM-02-75 [Figure 1a], are 
consistent with those reported in a previous study. ${ }^{16,21}$ The long flexible carbon chains of QM-02-75 were experimentally examined whether they demonstrate the existence of DFCT easily in this study. Moreover, another bis(azo) compound, QM-02-77 [Figure 1b], was originally synthesized to improve the reverse photo-isomerization from cis-isomers to trans-isomers; ${ }^{21}$ as a result, the HTP is optically increased. QM-02-77 consists of two terminal rigid units connected to an axially chiral core via ester linkages. These two terminal rigid units were originally added to reduce the degrees of freedom and to increase the steric hindrance of the cis-isomers; this process was conducted to improve the recovery of isomers from a cis configuration to a trans configuration. In particular, the rigid restricted side chains of the synthesized QM-02-77 provide the functions to reduce the free energy of the prepared cholesteric LC and consequently achieve a stable state in our system. Their chemical structures revealed that QM-02-77 has a larger chirality than QM-02-75. With the substitutions of the long flexible alkoxy chain of QM-02-75 and the terminal rigid units of QM-02-77, the combination of these two bis(azo) chiral dopants doped into nematic LCs with a negative dielectric anisotropy can achieve electrically switchable and permanently bistable scattering mode light modulators as a result of the switching between the large and small domains of DFCT with inhomogeneously helical axes. To the best of our knowledge, this study is the first to report that these dopants can be used to improve the electro-optical properties of scattering mode LC devices based on DSM and to achieve permanent stabilization. This novel development can be used for future practical applications in industrial and scientific fields. 
(a)

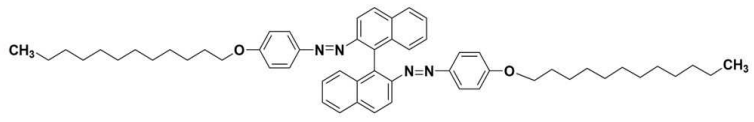

(b)

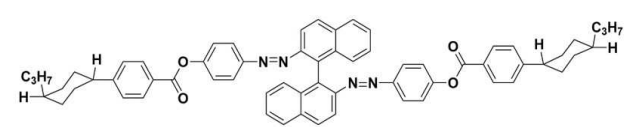

(c)

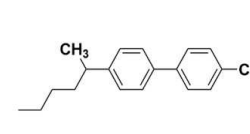

(d)

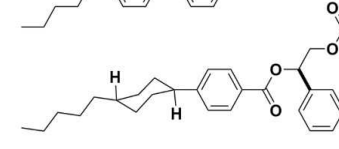

(e)

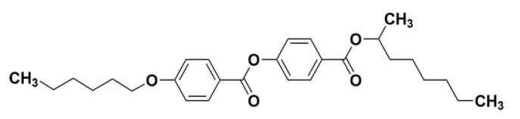

Figure 1. Molecular structures of the synthesized axially chiral bis(azo) dopants (a) QM-02-75 (QI-3c-S) and (b) QM-02-77; and the commercially chiral dopants (c) CB15, (d) R1011 and (e) S811.

\section{Experiments}

2.1 Preparation of Materials. After optimization was completed, the synthesized chiral dopants of 0.56 wt\% QM-02-75 (right-handed chirality, HTP $\sim 60 \mu \mathrm{m}^{-1}$ ) and 0.28 wt\% QM-02-77 (right-handed chirality, HTP $\sim 89 \mu \mathrm{m}^{-1}$ ) were doped into 99.16 wt\% commercially nematic LC, HNG30400-200 (clearing temperature $\sim 94{ }^{\circ} \mathrm{C}, \mathrm{n}_{\mathrm{e}} \sim 1.633, \mathrm{n}_{0} \sim 1.484, \Delta \varepsilon \sim-8.3$, FUSOL MATERIAL) to demonstrate the permanent stabilization of electrically switchable light scattering mode LC light modulators based on DFCT. The long-pitch-length cholesteric LC, whose pitch length was calculated to be $\sim 1.7 \mu \mathrm{m}$, provided low twisting power for the used materials and their reflection band located in the infrared region. The optimization parameters were determined by changing the concentrations of QM-02-75 and QM-02-77 to achieve the lowest visible light absorption ${ }^{21}$ (light 
yellowish color) and the best duration of stabilization after the applied voltage was switched off. Furthermore, the electro-optical properties of the materials of single azobenzene- and non-azobenzene chiral-doped HNG30400-200 were individually examined. The selected chiral dopants included QM-02-75, QM-02-77, QI-3c-S (left-handed chirality, purchased from BEAM Corp., Figure 1a, HTP -60 $\mu \mathrm{m}^{-1}$ ), CB15 (right-handed chirality, acquired from Merck, Figure 1c, HTP 7 $\mu \mathrm{m}^{-1}$ ), R1011 (right-handed chirality, purchased from Merck, Figure 1d, HTP 33 $\mu \mathrm{m}^{-1}$ ), and S811 (left-handed chirality, acquired from Merck, Figure 1e, HTP -11 $\left.\mu \mathrm{m}^{-1}\right)$. Among them, the chemical structures of QM-02-75, CB15, R1011, and S811 possess relative long flexible side chains (the shortest one is CB15), and that of QM-02-77 has terminal rigid restricted units. Interestingly, the chemical structures of QM-02-75 (synthesized in-house) and Ql-3c-S (commercial) are identical (Figure 1a); the key differences are the differences in the handedness of chiralities and the purities resulting from the course of reaction, of these two chiral azobenzenes. It is experimentally examined that single chiral (all six chiral dopants)-doped HNG30400-200 cannot show permanently stable scattering state when the applied voltage is switched off. Thus, the combination of QM-02-75 and QM-02-77 with the optimized concentration plays a key role in this study. The details of the optimization are presented in Section 3.3.

2.2 Fabrication of Cells. The inner surfaces of two indium-tin-oxide (ITO)-coated glass substrates were treated with homeotropic alignment films to obtain a scattering mode light modulator based on DFCT. An aqueous solution of $1.5 \%$ by volume dimethyloctadecy|[3-(trimethoxysilyl)propyl]ammonium chloride 
(DMOAP; anchoring energy $\sim 10^{-3}-10^{-2} \mathrm{~J} / \mathrm{m}^{2}$, purchased from Aldrich) was coated onto the inner surfaces of these two ITO-coated glass substrates by immersion-coating. ${ }^{24}$ Afterward, the coated DMOAP film was baked at $100^{\circ} \mathrm{C}$ for $1 \mathrm{~h}$ to generate a homeotropic alignment film. No rubbing treatment was required. Two DMOAP-coated substrates were assembled with $20 \mu \mathrm{m}$ cell gap to fabricate an empty cell. The homogeneously optimized sample was injected into the empty cell by capillary action at room temperature to prepare the scattering mode LC light modulator. The LC light modulator was then treated with an applied $\mathrm{AC}$ voltage of $40 \mathrm{~V}$ at $1 \mathrm{kHz}$ to switch to a transparent state. Thereafter, the electric switching of the LC light modulator was examined. Moreover, the alignment layer effect, including homogeneous (planar) alignment and hybrid alignment, on the electrically switchable scattering based on DFCT was also investigated. Experimentally, in addition to the absence of stable state in these systems, the required amplitudes of the applied DC/low-frequency AC electric field to present the scattering state of these two cases are higher than that of the LC cell with homeotropic alignment layers. This phenomenon suggests that the anchoring force parallel to the substrates resulting from the planar alignment layer is higher than that resulting from the homeotropic alignment layer; as such, the a relatively high electric field is required in these cases to induce ion transport for generating Williams' domains.

2.3 Measurements and Observations. The molecular structures of these two bis(azo) compounds were determined and confirmed by ${ }^{1} \mathrm{H}$ and ${ }^{13} \mathrm{C}$ nuclear magnetic resonance (NMR). The textures of the LC mixtures were observed 
under a polarized optical microscope (Olympus BX51TRF). For electro-optical measurement, the external applied voltages, including DC and AC signals, were generated by a function generator (RIGOL DG4102). The transmittance, response time, stabilization, and other properties of the scattering mode LC light modulator based on DFCT were obtained by using a He-Ne laser (Melles Griot, 5 $\mathrm{mW}$ ) and an oscilloscope (RIGOL DS4034). The photographs of the LC cells were taken by using a digital camera (Nikon D5300).

\section{Results and discussion}

\subsection{Electrical switching between transparent and scattering states.}

The curves depicted in Figure 2 present the variations in the stable transmittances of the scattering mode LC light modulator after an electric field with different frequencies (DC, 20, 60, 120, 150, and $200 \mathrm{~Hz}$ ) was applied. Each measurement was initially set in a transparent state. Notably, the initial transmittance of each datum was first reset to a transparent state by applying an AC voltage ( $40 \mathrm{~V}$ at $1 \mathrm{kHz}$ ); afterward, the electric field was applied and then was switched off to modulate the stable transmittance (Figure 2). The required amplitude of the applied voltages to switch the LC light modulator from a transparent state to a scattering state decreased as the frequency decreased. After the applied voltage was switched off, the scattering states presented by each point in each curve could be kept permanently stable. In the application of the $D C$ voltage, the optimized operating voltage was $0.3 \mathrm{~V} / \mu \mathrm{m}(\mathrm{DC})$, which was significantly lower than that described in previous studies. ${ }^{2-6}$ The images of the LC light modulator based on DFCT observed under a cross-polarizer polarized 
optical microscope (POM) in a transmissive mode are shown in the insets of Figure 2; the insets (a) and (b) depict the LC light modulator during the application of DC voltage (14 V, focal conic textures) and after the applied DC voltage was switched off (small domains of DFCT), respectively. The focal conic textures [inset (a) of Figure 2] can be observed because of the competition between the anchoring force perpendicular to the substrates provided by and the coated DMOAP films and the DC-induced ion transport and the anchoring force parallel to the substrates due to the negative dielectric anisotropy; as a result, the reorientation of the helical axis was disturbed to produce focal conic textures. After the applied voltage was switched off, the focal conic textures turned to small domains of DFCT [inset (b) of Figure 2], permanently stable scattering state due to the homeotropic alignment layer and the properties of the doped materials. The detailed mechanism will be given in Section 3.2.

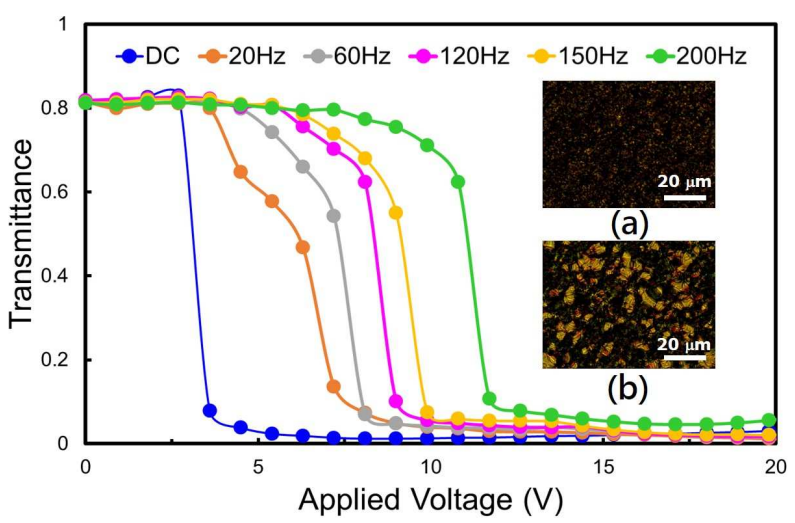

Figure 2. Variations in stable transmittance as functions of the applied voltage with different frequencies of LC light modulators from a transparent state to a scattering state. Insets show the images of the LC light modulator (a) during the application of DC voltage and (b) after the applied DC voltage (14 V) was switched off, as observed under a cross-polarizer polarized optical microscope in a transmissive mode. 
Reversely, the stable scattering states can be switched back to transparent states by applying an AC voltage of $>200 \mathrm{~Hz}$. Figure 3 shows the measured stable transmittance of the LC light modulator switching as a function of the applied AC voltages with different frequencies from scattering states to transparent states. All of the transmittances shown in these five curves were stable after the applied voltage was switched off. Each datum was obtained by applying an AC voltage to the LC light modulator treated with the applied DC voltage (14 V) to switch the LC cell to its initial highly scattering state. It indicates that the ions transported in these cases were reduced as the frequency of the applied voltage increased. The anchoring force parallel to the substrates resulting from the applied electric field caused the reorientation of the helical axes to the direction perpendicular to the substrates. The present textures, as shown in the inset (a) of Figure 3, during the application of an AC voltage (40 V at $1 \mathrm{kHz}$ ) were imperfect planar textures with large domains; hence, the textures exhibited transparency. After the applied voltage was removed, the cholesteric LC tended to switch from imperfect planar textures to large domains of DFCT [inset (b) of Figure 3], permanently stable transparent state due to the homeotropic alignment layer and the properties of the doped chiral materials. The detailed mechanism will be given in Section 3.2. The experimental results also reveal that if the selected frequency of the applied AC voltage is not sufficiently high enough to ignore the effect of ion transport, the required amplitude of the applied $A C$ voltage is relative high, as in the data shown in the curve of $400 \mathrm{~Hz}$ in Figure 3. Experimentally, the AC voltage with a frequency of $1 \mathrm{kHz}$ is an optimum candidate to switch the LC light modulator from 
a scattering state to a transparent state in this system. At $50 \mathrm{kHz}$, the curve of the stable transmittance versus the applied voltage is right shifted because of the reduction of the dielectric anisotropy $(\Delta \varepsilon)$ resulting in low anchoring force parallel to the substrate. Accordingly, the required amplitude of the applied voltages to switch the LC light modulator from the scattering state (small domains of DFCT) to the transparent state (large domains of DFCT) decreased as the frequency increased within the limits.

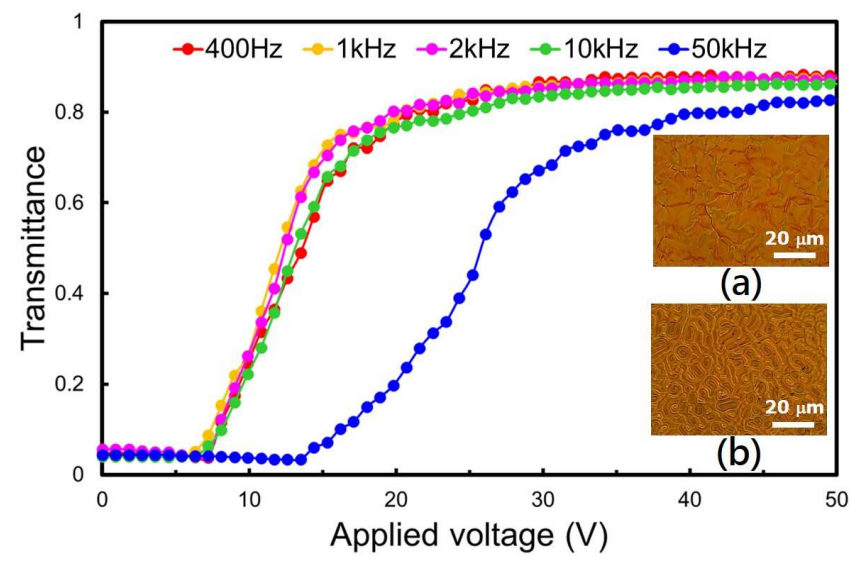

Figure 3. Variations in stable transmittance as functions of the applied voltage with different frequencies of LC light modulators from a scattering state to a transparent state. Insets show the images of the LC light modulator (a) during the application of $\mathrm{AC}$ voltage ( $40 \mathrm{~V}$ at $1 \mathrm{kHz}$ ) and (b) after the applied $\mathrm{AC}$ voltage was switched off, as observed under a cross-polarizer polarized optical microscope in a transmissive mode.

On the basis of our experimental results (Figures 2 and 3), we propose that the textures resulting in stable scattering and stable transparent states are small and large domains of DFCT with inhomogeneously helical axes, respectively. The domain size of the textures depicted in the inset (b) of Figure 2, is smaller than 
that shown in the inset (b) of Figure 3. When the stable transparent state is changed to the stable scattering state by applying a DC voltage, the domain size of the DFCT is significantly reduced to scatter light. Moreover, the selected pitch length of the cholesteric LC is extremely long; as such, the stable focal conic texture is absent in the system. ${ }^{25,26}$ The DMOAP alignment layer also provides a homeotropic anchoring to the LC molecules; this phenomenon indicates that the helical axes with a long-pitch cholesteric LC are aligned parallel to the substrates.

3.2 Mechanism of the electrical switching. Considering the mechanism of DSM for DFCT, we observed that the LC textures were reformed from large domains of DFCT [Figure 4a] to focal conic textures during electric field (DC- or low-frequency AC electric field) applications [Figure 4b]; this observation was attributed to the disturbance caused by the anchoring force perpendicular to the substrates resulting from the homeotropic alignment layer ( $A_{D M O A P}$ ) and the electric-field-induced ion-transport $\left(A_{\perp}\right)$, and the anchoring force parallel to the substrates resulting from the applied electric field $\left(A_{/ /}\right)$. In the generation of $A_{/ /}$ and $A_{\perp}$, the strength of $A_{\perp}$ depends on the amplitude of the applied electric field and on the physical properties of the adopted materials (e.g. viscosity, dielectric anisotropy, etc.). However, the strength of $A_{\perp}$ depends on the amplitude/frequency of the applied electric field and on the concentration of ions provided by the employed materials. Notably, the required time to switch the state from a transparent to a scattering one increased as the frequency of the applied voltage increased. If the strength of $A_{/ /}$and $A_{\perp}+A_{D M O A P}$ are comparable when a DC or low-frequency AC electric field is applied, these two anchoring do 
disturb the LC system to transfer the LC to scattering focal conic textures [Figure 4b]. After the applied voltage was switched off, the stable turbulent domains, or the so-called small domains of DFCT [Figure 4d], were generated to scatter light based on the Mie scattering. ${ }^{27}$ Conversely, the duration of the switching between positive and negative polarities of the applied voltage was shortened as the frequency of the applied voltage increased. In other words, with the application of the high-frequency $\mathrm{AC}$ electric field, the ions did not have sufficient time to transport in the bulk of the LC cell. In view of the dominant anchoring force parallel to the substrates, the LC textures changed to imperfect planar textures during the application of the high-frequency AC electric field [Figure 4c]. These findings suggest that if $A_{/ /}$is higher than $A_{\perp}+A_{D M O A P}$ when an electric field is applied, the anchoring force, $\mathrm{A}_{/ / \text {, }}$ dominates the LC system, thereby rotating LCs to become parallel to the substrates. Ultimately, both the large and small domains of DFCT can be transformed to imperfect planar textures (no perfect planar can be obtained because of the DMOAP alignment layer). Furthermore, after the applied voltage was switched off, the stable transparent domains, or the so-called large domain DFCT, were eventually formed [Figure 4a]. 
(a) Stable large domains of DFCT

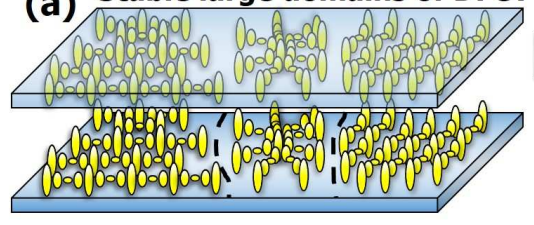

$\mathbf{A}_{/ /} \sim \mathbf{A}_{\perp}+\mathbf{A}_{\text {DMOAP }}$ DC field
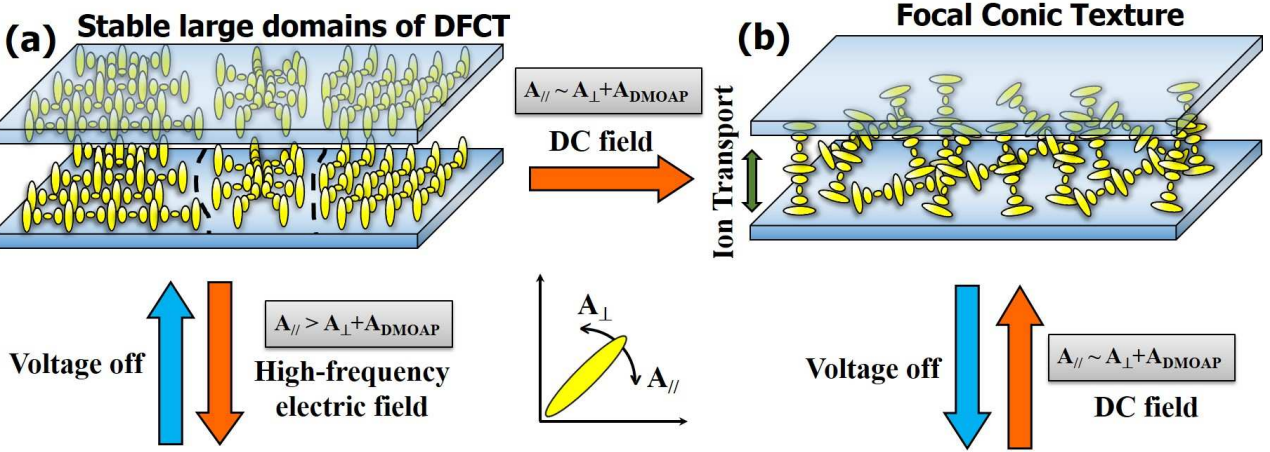

(c)

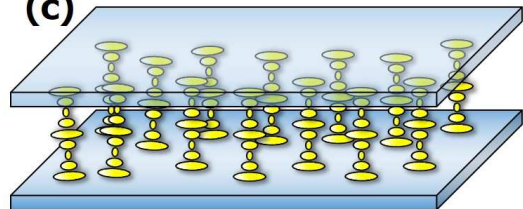

Imperfect Planar Texture

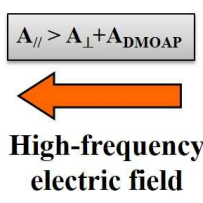

(d)

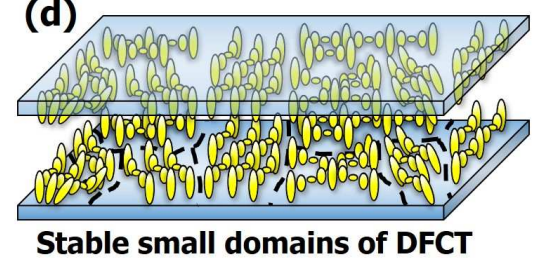

Stable small domains of DFCT

Figure 4. Mechanism of the electrically switchable light scattering by DFCT of (a) stably transparent large domains of DFCT; (b) scattering focal conic textures during DC/low-frequency AC electric field is applied; (c) transparent imperfect planar textures during high-frequency $A C$ electric field is applied; (d) stable scattering small domains of DFCT. Stable states mean that no external voltage is applied. $A_{/ /}$represents the anchoring parallel to the substrates resulting from the applied electric field. $A_{\perp}$ and $A_{D M O A P}$ depict the anchoring perpendicular to the substrates resulting from the electric-field-induced ion-transport and homeotropic alignment layer, respectively.

\subsection{Stabilization of the large and small domains of DFCT, and} optimization. The observations and examinations of the electro-optical properties were first elucidated by using a single-chiral-doped HNG30400-200. The selected chiral dopants include QM-02-75, QM-02-77, QI-3c-S, CB15, R1011， and S811, whose chemical structures are shown in Figure 1. The pitch lengths of these cholesteric LC were set to $\sim 1.7 \mu \mathrm{m}$ by tuning the concentration of each chiral dopant according to their HTP values, depending on the temperature and 
the LC host. Samples A-F in Table 1 show the experimental results of the transformation of various LC textures and their durations of stable time at scattering state after the applied DC voltage (14 V) was switched off. With the application of DC voltage, all six cholesteric LCs presented scattering focal conic textures. After the applied DC voltage was switched off, in addition to the QM-02-77-doped HNG30400-200, the other five cholesteric LCs were transformed to small domains of DFCT (scattering state), and slowly returned to large domains of DFCT (transparent state) after several minutes/hours, indicating that all of them (Samples A-F) do not possess permanent stable scattering state. Moreover, QM-02-77-doped HNG30400-200 shows no large and small domains of DFCT, and it showed a 30-minute scattering focal conic textures after the applied field was removed. Eventually, its stable state exhibits an imperfect planar texture.

Table 1. Descriptions of the LC light modulators fabricated by various mixtures of LC and chiral dopants after the applied DC voltages (14 V) was switched off.

\begin{tabular}{|c|c|c|c|c|c|}
\hline Sample & $\begin{array}{l}\text { Nematic } \\
\text { LCs }\end{array}$ & $\begin{array}{c}\text { Chiral } \\
\text { dopant A }\end{array}$ & $\begin{array}{l}\text { Chiral } \\
\text { dopant B }\end{array}$ & $\begin{array}{l}\text { Weight } \\
\text { concentration } \\
\text { ratio }\end{array}$ & $\begin{array}{l}\text { Description of the LC light modulator after the } \\
\text { applied DC voltages was switched off. } \\
\text { (DC 14 V, pitch length } \sim 1.7 \mu \mathrm{m} \text { ) }\end{array}$ \\
\hline $\mathbf{A}$ & \multirow{12}{*}{$\begin{array}{l}\text { HNG30400-200 } \\
\qquad \varepsilon \sim-8.3\end{array}$} & QM-02-75 & & & Slowly transformed to large domain DFCT within $\sim 5$ minutes. \\
\hline B & & QM-02-77 & & & $\begin{array}{l}\text { No DFCT, stable at focal conic textures for } \sim 30 \text { minutes, and } \\
\text { slowly transformed to imperfect planar textures. }\end{array}$ \\
\hline C & & QI-3c-S & & & Slowly transformed to large domain DFCT within $\sim 15$ minutes. \\
\hline D & & CB15 & & & $\begin{array}{l}\text { Stable at small domains of DFCT for }>2 \text { hours, and slowly } \\
\text { transformed to large domains of DFCT. }\end{array}$ \\
\hline $\mathbf{E}$ & & R1011 & & & Slowly transformed to large domain DFCT within $\sim 10$ minutes. \\
\hline $\mathbf{F}$ & & S811 & & & $\begin{array}{l}\text { Stable at small domains of DFCT for }>2 \text { hours and slowly } \\
\text { transformed to large domains of DFCT. }\end{array}$ \\
\hline G & & R1011 & QM-02-77 & $A: B=5: 1$ & $\begin{array}{l}\text { Stable at small domains of DFCT for }>2 \text { hours, and slowly } \\
\text { transformed to large domains of DFCT. }\end{array}$ \\
\hline $\mathbf{H}$ & & QM-02-75 & QM-02-77 & $A: B=5: 1$ & Slowly transformed to large domain DFCT within $\sim 10$ minutes. \\
\hline $\mathbf{I}$ & & QM-02-75 & QM-02-77 & $A: B=2: 1$ & Permanently stable at small domains of DFCT. \\
\hline J & & QM-02-75 & QM-02-77 & $A: B=1: 5$ & Transformed to large domain DFCT within a few seconds. \\
\hline $\mathbf{K}$ & & QM-02-75 & QM-02-77 & $A: B=1: 2$ & $\begin{array}{l}\text { Stable at small domains of DFCT for }>2 \text { hours, and slowly } \\
\text { transformed to large domains of DFCT. }\end{array}$ \\
\hline $\mathbf{L}$ & & QM-02-75 & QM-02-77 & $A: B=1: 1$ & $\begin{array}{l}\text { Stable at small domains of DFCT for }>2 \text { hours, and slowly } \\
\text { transformed to large domains of DFCT. }\end{array}$ \\
\hline
\end{tabular}


Figure 5 shows the observations for each single-chiral-doped HNG30400-200 (1) before / (2) during / (3) after the application of DC voltage (14 V) under a cross-polarizer POM. Columns (a)-(f) in Figure 5 depict the images of QM-02-75-, QM-02-77-, Ql-3C-S-, CB15-, R1011-, and S811-doped HNG30400-200, respectively. Figures $5 a-1,5 c-1,5 d-1,5 e-1$, and $5 f-1$ show large domains of DFCTs (transparent state), whereas only Figure 5b-1 presents an imperfect planar texture (transparent state). These experimental results shown in Table 1 and Figure 5 indicate that if the large domains of DFCT can be obtained by doping chiral dopant into nematics, such a large domain of DFCT is an intrinsically stable state (transparent state). For a sample of QM-02-77-doped HNG30400-200, the stable state presents an imperfect planar texture, but not large domains of DFCT. Furthermore, in view of the identical structures (except for the handedness of chiralities and the purities resulting from the course of reaction) of QM-02-75 and QI-3c-S, the observed images, shown in columns (a) and (c) in Figure 5, are extremely similar with each other. Row (2) in Figure 5 shows the textures of these samples during the application of DC voltage (14 V), and all of them demonstrate scattering focal conic textures. Figures $5 a-3,5 c-3,5 d-3,5 e-3$, and $5 f-3$ are small domains of DFCT after the applied DC voltages was switched off, and are all transient states. The durations of each stable time at small domains of DFCT can be read from Table 1. Eventually, all small domains of DFCT transform back to their initially transparent states [Row (1)], which are large domains of DFCT. 
(a)

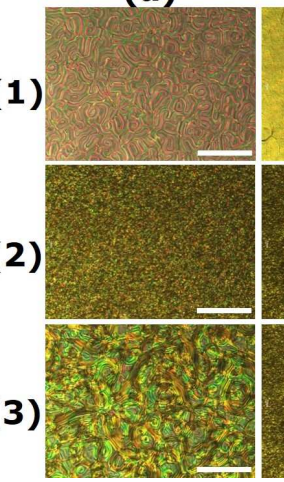

(b)

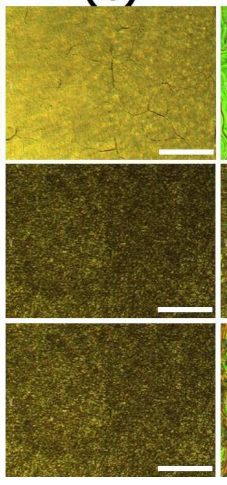

(c)

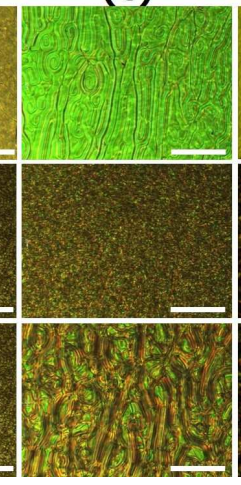

(d)

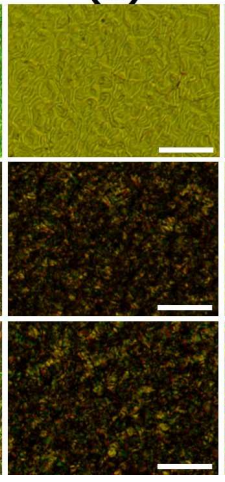

(e)

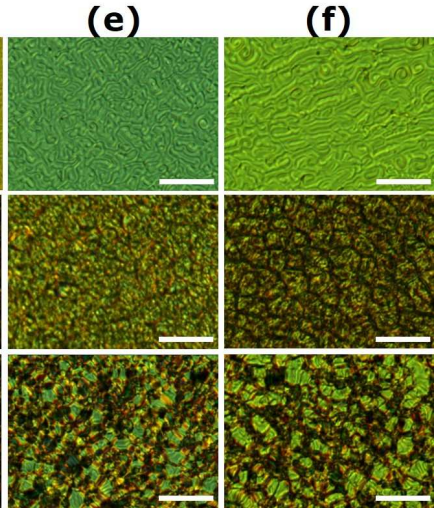

Figure 5. Images of the LC light modulators filled with (a) QM-02-75-, (b) QM-02-77-, (c) Ql-3c-S-, (d) CB15-, (e) R1011, and (f) S811-doped HNG30400-200, as observed under a cross-polarizer polarized optical microscope in transmissive mode. Rows represent the LC light modulator (1) at stable states, (2) during the application of DC voltage (14 V), and (3) after the applied DC voltage was switched off. The scale bars depict the length of $20 \mu \mathrm{m}$.

In consideration of the properties of our synthesized chiral dopants, QM-02-75 and QM-02-77, experimentally, QM-02-75-doped HNG30400-200 exhibited a high scattering focal conic state during DC voltage application. After the applied voltage was removed, the scattering small domains of DFCT (quasi-stable state) slowly transformed to transparent large domains of DFCT within 5 minutes (Sample A). Moreover, QM-02-77-doped HNG30400-200 showed a scattering focal conic texture during DC voltage application and maintained a stable scattering focal conic state for approximately 30 minutes (quasi-stable state) after the applied field was removed. Consequently, the focal conic textures naturally reverted to transparently imperfect planar textures but not to large domains of DFCT because of the chemical structures of the terminal rigid units (Sample B). It is believed that the terminal rigid units decline the free 
energy of the system and increase the energy barrier between the focal conic and planar textures to decelerate the transformation back to imperfect planar textures. With regard to the energy barrier, increased by the doped QM-02-77, the durations of stable time of R1011-doped- and (R1011+QM-02-77)-doped HNG30400-200 at small domains of DFCT were examined to be about 10 minutes (Sample E) and longer than 2 hours (Sample G), respectively. It indicates that the terminal rigid units of the doped small amount of QM-02-77 can also increase the energy barrier between the small and large domains of DFCT. Moreover, the experimental results of the commercial QI-3c-S-doped HNG30400-200 are similar to those of QM-02-75-doped HNG30400-200, except for the longer duration of stable time (quasi-stable state, $\sim 15$ minutes, Sample C) of the Ql-3c-S-doped HNG30400-200 at small domains of DFCT. The differences in their properties are caused by the differences in handedness of chirality and the purity as a result of the application of different synthesis processes.

With regard to the stabilization of the small domains of DFCT, our experimental results, as shown in Table 1 (Samples A, $\mathrm{H}$ and I), indicate that the duration of stable time of QM-02-75-doped HNG30400-200 at small domains of DFCT is only 5 minutes without the doping of the addition QM-02-77 (Sample A). The durations can be extended to about 10 minutes with the increase in the concentration of QM-02-77 (Sample H). Moreover, the small domains of DFCT become permanently stable with the doping of the optimized concentration of QM-02-77 (Sample I). However, if the concentration of QM-02-77 was set to even higher values (Samples J-L), the durations of stable time at small domains of 
DFCT became shorter and shorter. As described above, QM-02-77-doped HNG30400-200 presents no fingerprint textures, and does present imperfect planar textures. The experimental results, shown in Samples B and J-L in Table 1, show that the DFCT can be generated with the increase of the concentration of QM-02-75. The durations of stable time at small domains of DFCT can be extended to the range between a few second (Sample J) and longer than 2 hours (Sample L). In summary of QM-02-75 and QM-02-77, the role of the doped QM-02-77 herein is to reduce the free energy of the DFCT system, and prefers to be stable at imperfect planar texture. Furthermore, to generate scattering mode based on small domains of DFCT, a small amount of QM-02-75 should be added into QM-02-77-doped HNG30400-200 to initiate the formation of DFCT. Therefore, an optimized weight concentration ratio of QM-02-75 and QM-02-77 that provides the low enough free energy to maintain the permanently small domains of DFCT can be obtained. In this paper, the optimized concentration of these two dopants (QM-02-75: QM-02-77) was found to be 2:1 for the permanently stable scattering modulator. The combination of the functions of QM-02-75 and QM-02-77 can improve the performances of these materials in bistable light scattering modes.

3.4 Electro-optical properties of the light modulator. Switching times of LC light modulator from transparent to scattering states by the application of various DC/AC voltages were measured, as plotted in Figure 6a. The switching time decreased as the applied voltage increased. The disturbance from the ion transport and the anchoring force caused by the applied voltage could be 
exacerbated as the applied voltage increased. Hence, the reformation rate of DFCT could be increased to reduce the switching time. According to the experimental results, the switching time from a transparent state to a scattering state at a DC voltage of $14 \mathrm{~V}(0.7 \mathrm{~V} / \mu \mathrm{m})$ was approximately $10 \mathrm{~ms}$, as shown in the inset of Figure 6a. Conversely, the required switching time from the scattering state to the transparent state by the application of various high-frequency $\mathrm{AC}$ electric fields was considered, as plotted in Figure $6 \mathrm{~b}$. The required time decreased as the applied voltage increased. According to the experimental results, the switching time from the scattering state to the transparent state by applying an AC voltage [40 V (2 V/ $\mu \mathrm{m})$ at $1 \mathrm{kHz}$ ] was approximately $450 \mathrm{~ms}$. Unfortunately, the switching time is too long to be applied to real-time displays; however, the switching time is suitable for the applications of bistable displays, such as e-paper, e-book, and other electronics. A separate experiment, as shown in the inset of Figure $6 \mathrm{~b}$, shown that with the application of $\mathrm{AC}$ voltage of $4 \mathrm{~V} / \mu \mathrm{m}$ at $1 \mathrm{kHz}$, the switching time can be reduced to approximately 45 ms. 


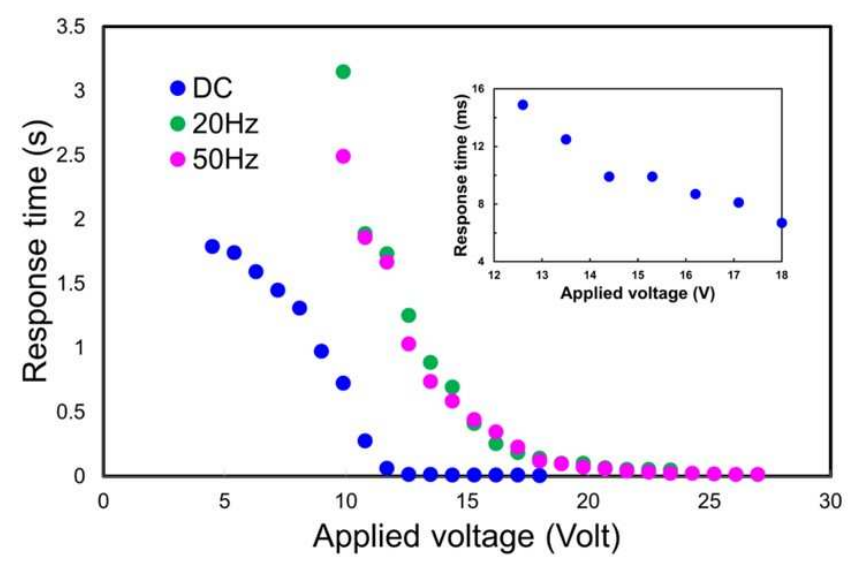

(a)

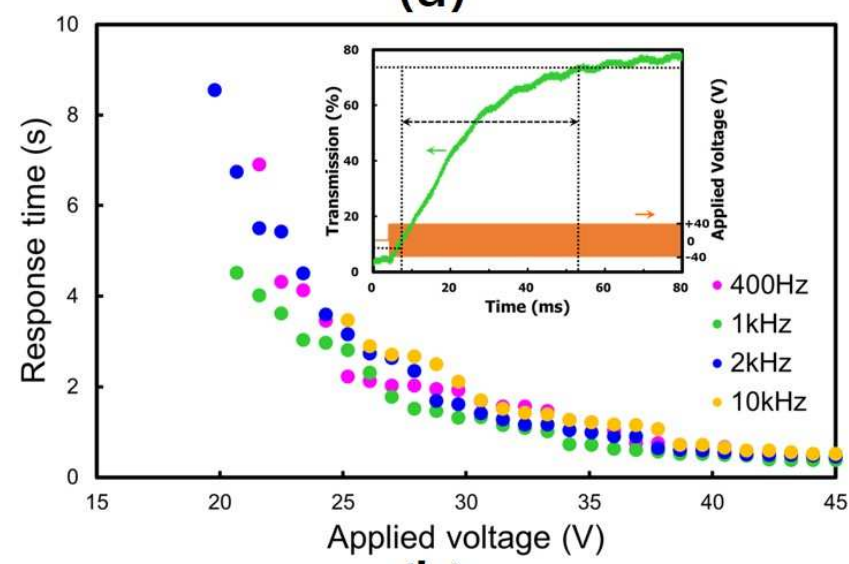

(b)

Figure 6. Response time (a) from a transparent state to a scattering state versus applied voltage (DC and $A C 20 \mathrm{~Hz}$ ) and (b) from a scattering state to a transparent state versus applied voltage with different frequencies of LC light modulators. Inset of (a) represents the details of the response time resulting from the application of DC voltage between 12 and $18 \mathrm{~V}$. Inset of (b) shows the switching response ( $45 \mathrm{~ms}$ ) of LC light modulator from a scattering state to a transparent state by the application of $A C$ voltage of $80 \mathrm{~V}(4 \mathrm{~V} / \mu \mathrm{m})$ at $1 \mathrm{kHz}$.

The frequency effect onto the switching of the scattering mode LC light modulator was discussed. The amplitude of the applied voltage was selected as $20 \mathrm{~V}$. The variations in the stable transmittance as functions of the frequency of 
the applied voltage were determined. Curves (a) and (b) in Figure 7 respectively plot the variations in the stable transmittance after the electric field with different frequencies was applied to the LC light modulator from the scattering state to the transparent state and vice versa. Consistent with the measurement in Figures 2, 3 and 6, the stable transmittances in Curves (a) and (b) were reset to their initial scattering (small domains of DFCT) and transparent (large domains of DFCT) states by respectively applying $\mathrm{DC}(14 \mathrm{~V})$ and $\mathrm{AC}(40 \mathrm{~V}$ at $1 \mathrm{kHz})$ voltages before the electric field was applied. In addition to the switching achieved by modulating the amplitude of the applied field, the grayscales can also be obtained by modulating the frequency of the applied electric field. In brief, the required anchoring force parallel to the substrate provided by the applied voltage to initiate the orientation of LC from the scattering state with small domains of DFCT should be larger than that from the transparent state with large domains of DFCT. Moreover, the effective ion transport that provided the anchoring force perpendicular to the substrate decreased as the frequency of the applied voltage increased and vice versa. Hence, the effective anchoring force caused by the applied voltage and the ion transport determined the difference in the transmittance between these two initial states. 


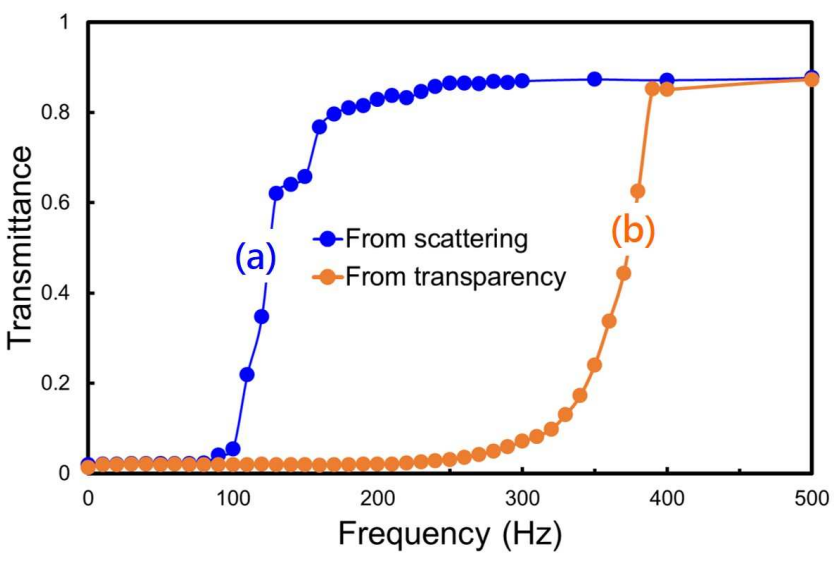

Figure 7. Variations in stable transmittance as functions of the frequency of the applied electric field of the LC light modulator from (a) a scattering state and (b) a transparent state.

Figures $8 a-8 c$ show the photographs of the scattering mode LC light modulator at transparent, one grayscale, and scattering states, respectively. These three states are stable. Figure $8 \mathrm{a}$ shows the transparent state with a transmittance of about $80 \%$ obtained by applying an AC voltage of $40 \mathrm{~V}$ at $1 \mathrm{kHz}$. The cause of the low initial transmittance, as shown in Figure 8a, include the visible light absorption by doped azobenzene and the scattering resulting from the some relative small domains, comparing with the large domains of DFCT (Figure 3b). Figures $8 b(8 c)$ depicts one grayscale (scattering) state with a transmittance of about $33 \%(0.5 \%)$ obtained by applying the DC voltage from the transparent state (Figure 8a). The contrast ratio of the LC light modulator is defined as the ratio of the maximum transmittance to the minimum transmittance; in this study, the maximum contrast ratio of the LC light modulator was calculated as 200 according to Figure 2. 
Comparing the electro-optical properties of the other light scattering mode LC light modulators, including the dynamic scattering mode, ${ }^{2-3} \mathrm{SmA}^{5,9}$ organosiloxane $\mathrm{LCs}^{6}{ }^{6}$ polymer-dispersed bistable $\mathrm{SmA},{ }^{10}$ dye-doped PDLCs, ${ }^{11}$ particular thermal induced phase separation, ${ }^{12}$ supramolecular LC gels, ${ }^{13}$ dual frequency LCs, ${ }^{14-15}$ and others, with DFCT reported in this study, their properties of bistability, operating voltage, and contrast are listed in Table 2. The operating voltage of $\mathrm{T}$ to $\mathrm{S}$ (S to $\mathrm{T}$ ) represents the required voltage for switching the $\mathrm{LC}$ light shutter from highest transmission (scattering) to the highest scattering (transmission).

Table 2. Comparisons of the electro-optical properties of various kinds of scattering mode LC light modulators.

\begin{tabular}{|c|c|c|c|c|}
\hline \multirow{2}{*}{ Types } & \multirow{2}{*}{ Bistability } & \multicolumn{2}{|c|}{ Operating voltage* } & \multirow{2}{*}{ Contrast } \\
\hline & & $\mathbf{T}$ to $\mathbf{S}$ & $\mathbf{S}$ to $\mathbf{T}$ & \\
\hline Dynamic Fingerprint Chiral Textures & Permanent & DC 7 V & $25 \mathrm{~V} @ 1 \mathrm{KHz}$ & 200 \\
\hline Dynamic Scattering: Nematic LCs [2] & No & DC $70 \mathrm{~V}$ & $\mathbf{v}_{\text {off }}$ & 20 \\
\hline Dynamic Scattering: Mixed LCs [3] & Several hours & DC $36 \mathrm{~V}$ & >100 V@1 KHz & 7 \\
\hline Smectic A LCs [5] & Permanent & $80 \mathrm{~V} @ 100 \mathrm{~Hz}$ & $40 \mathrm{~V} @ 1 \mathrm{KHz}$ & $<10$ \\
\hline Organosiloxane LCs [6] & Permanent & $80 \mathrm{~V} @ 100 \mathrm{~Hz}$ & 200 V@3 KHz & $* *$ \\
\hline Smectic A LCs [9] & Permanent & 80 V@50 Hz & $80 \mathrm{~V} @ 5 \mathrm{KHz}$ & $* *$ \\
\hline Polymer-Dispersed Bistable Smectic A LCs [10] & Permanent & $45 \mathrm{~V} @ 50 \mathrm{~Hz}$ & 72 V @5 KHz & $<5$ \\
\hline Dye-Doped Polymer Dispersed LCs [11] & No & $\mathbf{V}_{\text {off }}$ & $5 \mathrm{~V} @ 1 \mathrm{KHz}$ & $<45$ \\
\hline Particular TIPS [12] & No & $\mathbf{V}_{\text {off }}$ & $18 \mathrm{~V} @ 1 \mathrm{KHz}$ & $>300$ \\
\hline Supramolecular LC Gels [13] & No & $\mathbf{V}_{\text {off }}$ & $2.7 \mathrm{~V} @ 1 \mathrm{KHz}$ & $>1000$ \\
\hline Dual Frequency Cholesteric LCs [14] & Permanent & 20 V@1 KHz & $20 \mathrm{~V} @ 100 \mathrm{KHz}$ & $<50$ \\
\hline Dual Frequency Cholesteric LCs [15] & Permanent & $30 \mathrm{~V} @ 1 \mathrm{KHz}$ & $30 \mathrm{~V} @ 50 \mathrm{KHz}$ & $<6$ \\
\hline
\end{tabular}

*Operating voltage: $\mathbf{T}$ and $\mathbf{S}$ mean the highest transmission and scattering, respectively.

$* *$ Contrasts in these cases cannot be obtained from the corresponding references.

Moreover, considering the isomerization of the doped azobenzenes, we demonstrate in a separate experiment, as shown in Figure 9, that the photo-isomerization effect of the doped chiral azobenzenes as a result of the variation in the pitch length of the used cholesteric LCs does not affect the performance of the LC light modulator, including bistability, DC/AC switching, and scattering performance. ${ }^{16-21}$ Briefly, Figures 9a and 9e show the photograph and 
image observed under a cross-polarizer POM, respectively, of a LC light modulator after being treated with $\mathrm{AC}$ voltage ( $40 \mathrm{~V}$ at $1 \mathrm{KHz}$ ) to switch the textures to transparent large domains of DFCT (transmittance 80.37\%, trans-isomers dominant). After which, the LC light modulator was illuminated with UV light to initiate photo-isomerization. At transparent state, the measured transmittance of the LC cell having large domains of DFCT was almost invariant (transmittance 80.11\%, cis-isomers dominant). The observations are shown as Figures $9 \mathrm{~b}$ and $9 \mathrm{f}$. Thereafter, the LC cell was treated with thermal process $\left(80^{\circ} \mathrm{C}\right.$ for 1 minute) to isomerize the isomers from cis to trans states, ${ }^{25}$ and with DC voltage $(14 \mathrm{~V})$ to switch the textures to small domains of DFCT (scattering state). After the experiments described above were repeated, the transmittances of the LC cell at scattering state before (Figures $9 \mathrm{c}$ and $9 \mathrm{~g}$, trans-isomers dominant) and after (Figures 9d and 9h, cis-isomers dominant) the treatment of UV illumination were approximately $0.417 \%$ and $0.423 \%$, respectively. The LC light modulator before and after being treated with UV illumination was almost unaffected, except for the changes of the DFCT domain size and the pitch length, which did not reduce the transmission and scattering performance according to the experimental measurement. Accordingly, these results suggest that the performance is unaffected by photoisomerization effect because of the very small amount of the required azobenzenes. 


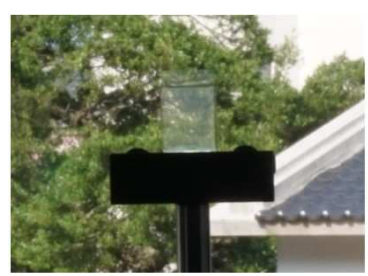

(a)

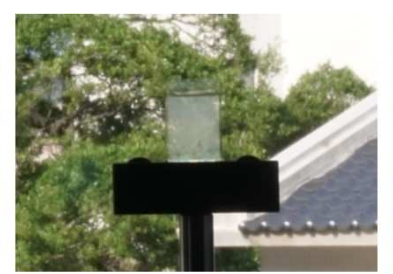

(b)

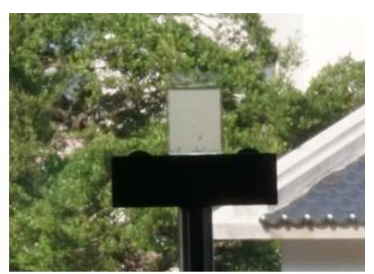

(c)

Figure 8. Photographs of the LC light modulator of (a) a transparent state (transmittance of approximately 80\%), (b) one gray scale (transmittance of approximately $33 \%$ ), and (c) a scattering state (transmittance of approximately $0.5 \%)$.

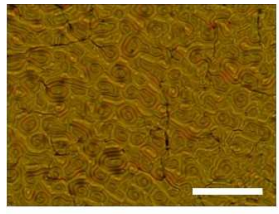

(a)

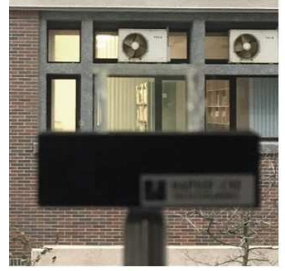

(e)

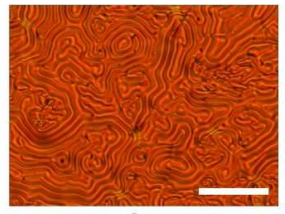

(b)

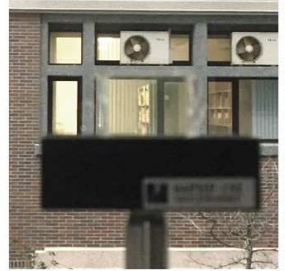

(f)

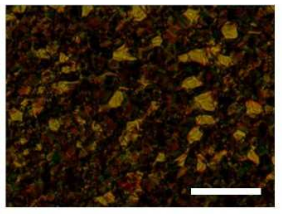

(c)

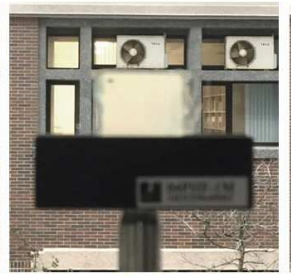

(g)

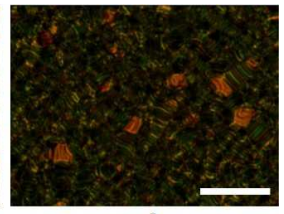

(d)

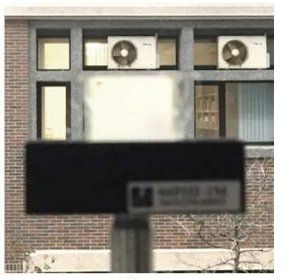

(h)

Figure 9. Images of the LC light modulator at transparent [scattering] state (a) [(c)] before (trans-isomer dominant) and (b) [(d)] after (cis-isomer dominant) the treatment of UV illumination, as observed under a cross-polarizer polarized optical microscope in a transmissive mode. The scale bars depict the length of 20 $\mu \mathrm{m}$. Photographs (e)-(h) are the LC scattering mode light modulators of images (a)-(d).

With regarding the transparency (scattering) performance of the large (small) domains of DFCT, the circles and triangles in Figure 10a denote the plots of the measured transmission of such a LC light modulator in transparent and scattering 
states, respectively, as a function of the polarization state of incident light. These results show that both transparency and scattering are independent of polarization, and that the averages of the measured transmission in transparent (larger domains of DFCT) and scattering (small domains of DFCT) states are approximately $80 \%$ and $0.4 \%$, respectively. The reason for the polarization independence is that the helical axes of the small and large domains of DFCT were inhomogeneously oriented, such that no optical anisotropy could be observed. Moreover, the viewing angle of the transparent state attributed to the large domains of DFCT is not limited by the viewing-angle-dependent refractive index of LCs, such as PDLC. ${ }^{28}$ Figures $10 \mathrm{~b}-10 \mathrm{~d}$ show that the observations of the bistable LC light modulator having transparent large domains of DFCT from different viewing angles, namely, $-70^{\circ}, 0^{\circ}$ and $70^{\circ}$. The proposed bistable light scattering mode clearly offers a wide viewing angle at both transparent and scattering states. The effective refractive indices of the large domains of DFCT from different incident angles are the same; thus, the observed refractive index is continuous. 


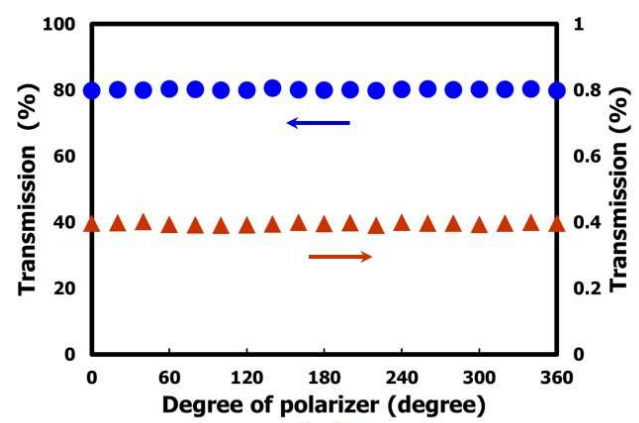

(a)

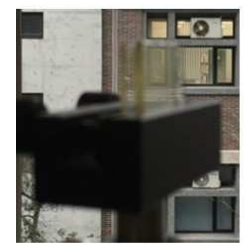

(b)

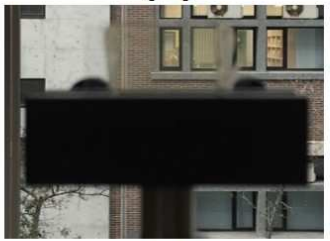

(c)

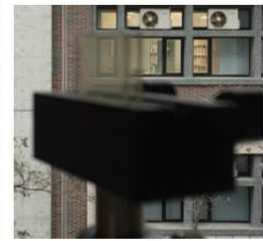

(d)

Figure 10. Circles and triangles in (a) show the plots of the measured transmission of such a LC light modulator in transparent and scattering states, respectively, as a function of polarization state of incident light. Photographs of bistable LC light modulator having transparent large domains of DFCT from different viewing angle of (b) $-70^{\circ}$; (c) $0^{\circ}$ and (d) $70^{\circ}$.

\section{Conclusion}

In conclusion, new azobenzene materials doped into nematic LCs with a negative dielectric anisotropy at low concentrations can be used to develop electrically switchable, permanently bistable, wide-viewing-angle, high-contrast, and low-operating-voltage, polarization independent scattering mode LC light modulators. Experimentally, the synthesized chiral dopant, QM-02-75, whose chemical structure contains a binaphthyl core linked with a long flexible alkoxy chain, doped into negative dielectric anisotropic nematics presents DFCT. The terminal rigid restricted side chains of QM-02-77 provide the stabilization of the small domains of DFCT in this system. The large domains of DFCT present a 
transparent state, and the small domains of DFCT cause a scattering state. In addition to permanent stabilization, the required amplitude of the applied voltage ( $A C$ and/or $D C$ ) is much lower than that of previously reported bistable scattering mode LC light modulator based on DSM. To the best of our knowledge, this study is the first to report the permanent stabilization and low operating voltage of scattering mode LC light modulators based on DFCT. Moreover, the required concentration of these two new azobenzene materials with the properties of trans-cis photoisomerization was optimized to be extremely low; as such, neither can affect the electro-optical behavior of light modulators. Such dopants can be considered an exciting impetus for promising practically potential for the optical applications of stable scattering mode LC devices with extremely low power consumption, high contrast, wide-viewing-angle, and so on. Considering the practical applications of LC devices, such as e-book and e-paper, the dynamic drive schemes should further be developed for such dynamic scattering mode LC light modulators based on DFCT. Furthermore, according to such interesting results, some further studies for the detailed discussion about the precisely stable durations of small domains of DFCT for each case, the voltage effect onto the bistable device, and others, are underway.

\section{Acknowledgement}

The authors would like to thank the Ministry of Science and Technology (MOST) of Taiwan for financially supporting this research under Grant No. MOST 103-2112-M-008-018-MY3. We are also grateful to Prof. Jy-Shan Hsu (Chung 
Yuan Christian University, Taiwan) for allowing full equipment usage. Importantly, we also sincerely thank the reviewers for their valuable comments and suggestions. 


\section{References}

(1) Blinov, L. M.; Chigrinov, V. G. Electrooptic Effects in Liquid Crystal Materials, Springer, 1996.

(2) Heilmeier, G. H.; Zanoni, L. A.; Barton, L. Dynamic Scattering: A New Electrooptic Effect in Certain Classes of Nematic Liquid Crystals. Proc. IEEE 1968, 56, 1162-1171.

(3) Heilmeier, G. H.; Goldmacher, J. E. A New Electric Field Controlled Reflective Optical Storage Effect in Mixed Liquid Crystal Systems. Proc. IEEE 1969, 57, 34-38.

(4) Coates, D.; Crossland, W. A.; Morrissy, J. H.; Needham, B. Electrically Induced Scattering Textures in Smectic A Phases and Their Electrical Reversal. J. Phys. D: Appl Phys. 1978, 11, 2025-2034.

(5) Gardiner, D. J.; Morris, S. M.; Coles, H. J. High-Efficiency Multistable Switchable Glazing Using Smectic A Liquid Crystals. Sol Energy Mater. Sol. Cells 2009, 93, 301-306.

(6) Gardiner, D. J.; Coles, H. J. Organosiloxane Liquid Crystals for Fast-Switching Bistable Scattering Devices. J. Phys. D: Appl Phys. 2006, 39, 4948-4955.

(7) Morris, S. M.; Gardiner, D. J.; Qasim, M. M.; Hands, P. J. W.; Wilkinson, T. D.; Coles, H. J. Lowering the Excitation Threshold of a Random Laser using the Dynamic Scattering States of an Organosiloxane Smectic A Liquid Crystal. J. App. Phys. 2012, 111, 033106.

(8) Khan, A. A.; Morris, S. M.; Gardiner, D. J.; Qasim, M. M.; Wilkinson, T. D.; Coles, H. J. Improving the Stability of Organosiloxane Smectic A Liquid Crystal Random Lasers using Redox Dopants. Optical Materials 2015, 42, 441-448. 
(9) Lu, Y.; Guo, J.; Wang, H.; Wei, J. Flexible Bistable Smectic-A Liquid Crystal Device Using Photolithography and Photoinduced Phase Separation. Adv. Cond. Matter. Phys. 2012, 2012, 843264. (doi:10.1155/2012/843264)

(10) Lu, Y.; Wei, J.; Shi, Y.; Jin, O.; Guo, J. Effects of Fabrication Condition on The Network Morphology and Electro-Optical Characteristics of Polymer-Dispersed Bistable Smectic A Liquid Crystal Device. Liq. Cryst. 2013, 40, 581-588.

(11) Yang, K. J.; Yoon, D. Y. Electro-Optical Characteristics of Dye-Doped Polymer Dispersed Liquid Crystals. J. Ind Eng. Chem. 2011, 17, 543-548.

(12) Chen, Y. D.; Fuh, A. Y. G.; Cheng, K. T. Particular Thermally Induced Phase Separation of Liquid Crystal and Poly(N-Vinyl Carbazole) Films and its Application. Opt. Express 2012, 20, 16777-16784.

(13) Chen, J. W.; Huang, C. C.; Chao, C. Y. Supramolecular Liquid-Crystal Gels Formed by Polyfluorene-Based $\pi$-Conjugated Polymer for Switchable Anisotropic Scattering Device. ACS Appl. Mater. Interfaces 2014, 6, 6757-6764.

(14) Hsiao, Y. C.; Tang, C. Y.; Lee, W. Fast-switching Bistable Cholesteric Intensity Modulator. Opt. Express 2011, 19, 9744-9749.

(15) Kumar, P.; Kang, S. W.; Lee, S. H. Advanced Bistable Cholesteric Light Shutter with Dual Frequency Nematic Liquid Crystal. Opt. Mat. Express 2012, 2, 1121-1134.

(16) Li, Q.; Green, L.; Venkataraman, N.; Shiyanovskaya, I.; Khan, A.; Urbas, A.; Doane, J. W. Reversible Photoswitchable Axially Chiral Dopants with High Helical Twisting Power. J. Am. Chem. Soc. 2007, 129, 12908-12909.

(17) White, T. J.; Cazzell, S. A.; Freer, A. S.; Yang, D. K.; Sukhomlinova, L.; Su, L.; Kosa, T.; Taheri, B.; Bunning, T. J. Widely Tunable, Photoinvertible Cholesteric Liquid 
Crystals. Adv. Mater. 2011, 23, 1389-1392.

(18) White, T. J.; Bricker, R. L.; Natarajan, L. V.; Tabiryan, N. V.; Green, L.; Li, Q.;

Bunning, T. J. Phototunable Azobenzene Cholesteric Liquid Crystals With 2000 nm Range. Adv. Funct. Mater. 2009, 19, 3484-3488.

(19) Li, Q.; Li, Y.; Ma, J.; Yang, D. K.; White, T. J.; Bunning, T. J. Directing Dynamic Control of Red, Green, and Blue Reflection Enabled by a Light-Driven Self-Organized Helical Superstructure. Adv. Mater. 2011, 23, 5069-5073.

(20) Lin, T. H.; Li, Y.; Wang, C. T.; Jau, H. C.; Chen, C. W.; Li, C. C.; Bisoyi, H. K.; Bunning, T. J.; Li, Q. Red, Green and Blue Reflections Enabled in an Optically Tunable Self-Organized 3D Cubic Nanostructured Thin Film. Adv. Mater. 2013, 25, 5050-5054.

(21) Morris, S. M.; Qasim, M.; Cheng, K. T.; Castles, F.; Ko, D. H.; Gardiner, D. J.; Nosheen, S.; Wilkinson, T. D.; Coles, H. J.; Burgess, C.; Lee, H. Optically Activated Shutter using a Photo-Tunable Short-Pitch Chiral Nematic Liquid Crystal. Appl. Phys. Lett. 2013, 103, 101105.

(22) Xie, Y.; Fu, D.; Jin, O.; Zhang, H.; Wei, J.; Guo, J. Photoswitchable Molecular Switches Featuring Both Axial and Tetrahedral Chirality. J. Mater. Chem. C, 2013, 1, 7346-7356.

(23) Guo, J.; Wang, J.; Zhang, J.; Shi, Y.; Wang, X.; Wei, J. Photo- and Thermal Switching of Blue Phase Films Reflecting Both Right- and Left-Circularly Polarized Light. J. Mater. Chem. C, 2014, 2, 9159-9166.

(24) Škarabot, M.; Ravnik, M.; Žumer, S.; Tkalec, U.; Poberaj, I.; Babič, D.; Osterman, N.; Muševič, I. Interactions of quadrupolar nematic colloids. Phys. Rev. E 2008, $77,031705$. 
(25) Geng, J.; Dong, C.; Zhang, L.; Ma, Z.; Shi, L.; Cao, H.; Yang, H. Electrically

Addressed and Thermally Erased Cholesteric Cells. Appl. Phys. Lett. 2006, 89, 081130.

(26) Fuh, A. Y. G.; Wu, Z. H.; Cheng, K. T.; Liu, C. K.; Chen, Y. D. Direct Optical Switching of Bistable Cholesteric Textures in Chiral Azobenzene-Doped Liquid Crystals. Opt. Express 2013, 21, 21840-21846.

(27) Marusii, T. Ya.; Reznikov, Yu. A.; Reshetnyak, V. Yu.; Soskin, M. S.; Khizhnyak, A. I. Scattering of Light by Nematic Liquid Crystals in Cells with a Finite Energy of the Anchoring of the Director to The Wails. Sov. Phys. JETP 1986, 64, 502-507.

(28) Wu, B. G.; West, J. L.; Doane, J. W. Angular discrimination of light transmission through polymer-dispersed liquid-crystal films. J. Appl. Phys. 1987, 65, 3925-3931.

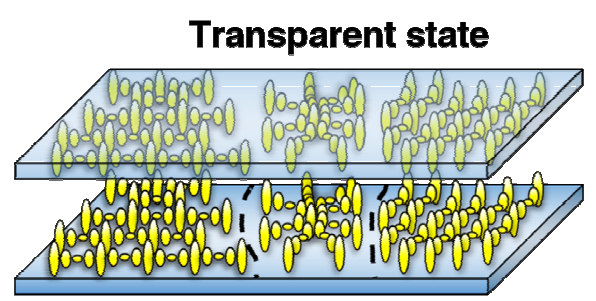

Stable large domains of DFCT
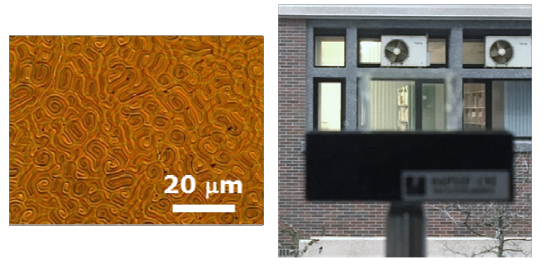

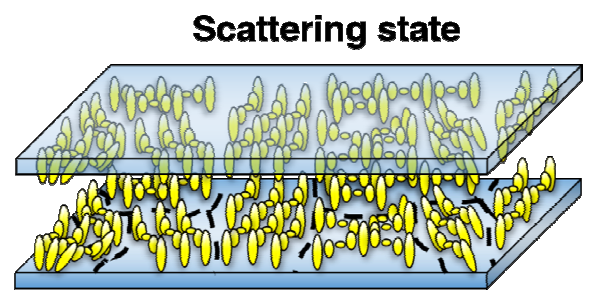

Stable small domains of DFCT
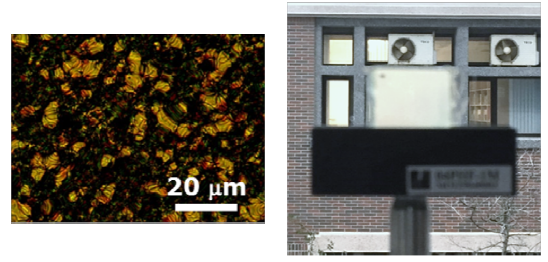

Table of Contents 